\title{
Vibrations of a deformed half-space with inclusion under the influence of surface waves
}

\author{
Bakhodir Rakhmonov ${ }^{10000-0002-2978-6890]}$, Ismoil Safarov ${ }^{* 2,3}$, Mukhsin Teshaev $^{3,4}$, and Ravshan Nafasov ${ }^{1}$ \\ ${ }^{1}$ Urgench State University, 220100 Urgench, Uzbekistan \\ ${ }^{2}$ Tashkent Institute of Chemical Technology, 100011 Tashkent, Uzbekistan \\ ${ }^{3}$ Bukhara Institute of Engineering and Technology, 200100 Bukhara, Uzbekistan, \\ ${ }^{4}$ Bukhara branch of the Tashkent Institute of Irrigation and Mechanization Engineering in Agricultural, \\ 105009 Bukhara, Uzbekistan
}

\begin{abstract}
There is a large number of underground tunnels of various shapes located in seismic zones that need to be protected from seismic impacts. The paper considers the effect of harmonic surface waves on a cylindrical inclusion of various shapes located in a viscoelastic half-plane. The main purpose of the study is to determine the stress-strain state of the obstacle when exposed to harmonic waves. The problem is solved by the finite element method. It was found that the maximum stress concentration is allowed at long waves, and the stress concentration with increasing depth and wavelength approaches the static value of stress. The reliability of the obtained research results is confirmed by good agreement with theoretical and experimental results obtained by other authors.
\end{abstract}

Keywords. Seismic waves, body vibrations, arch effect, Rayleigh wave, Gauss method, elastic medium, harmonic load.

\section{Introduction}

In the dynamic case, the arching effect has hardly been experimentally studied. The main published works are of a theoretical nature. A number of experimental studies were carried out in order to assess the distribution and effects of arches, it was found out that the pressure acting on the length of the rate does not depend on the structure of the stress distribution in the soil layer located above its surface at a distance of two or three of its width, the results of one of the experiments are the vertical component of pressure, $\sigma_{v}$ depth $Z=H$ without the effect of strength (i.e., hydrostatic load) was $\sigma_{v h}$. As can be seen from the figure, for a soil layer with a thickness of $Z<0.4\left(Z_{b}>2 s\right)$ on the stress distribution diagram, there is no decrease in the vertical component due to the absence of a durable effect. But for $Z=H$, i.e., in the area of the ground located outside the mediocre proximity to the rectangular insert, $\sigma_{v}$ is at least $10 \% \sigma_{v}$.

\section{Method}

\subsection{Statement of the problem and methods of solution}

We consider linear vibrations of an elastic half-space with obstacles (with different outlines) under the action of Rayleigh-type surface waves (Fig. 1). Let us associate the Cartesian

${ }^{*}$ Corresponding author: safarov54@mail.ru 
coordinate system OXYZ with the axis of the cylinder. The problem under consideration is reduced to the plane problem of the theory of elasticity (plane deformation). Let us investigate the effect of a Rayleigh surface wave with a cylindrical layer.

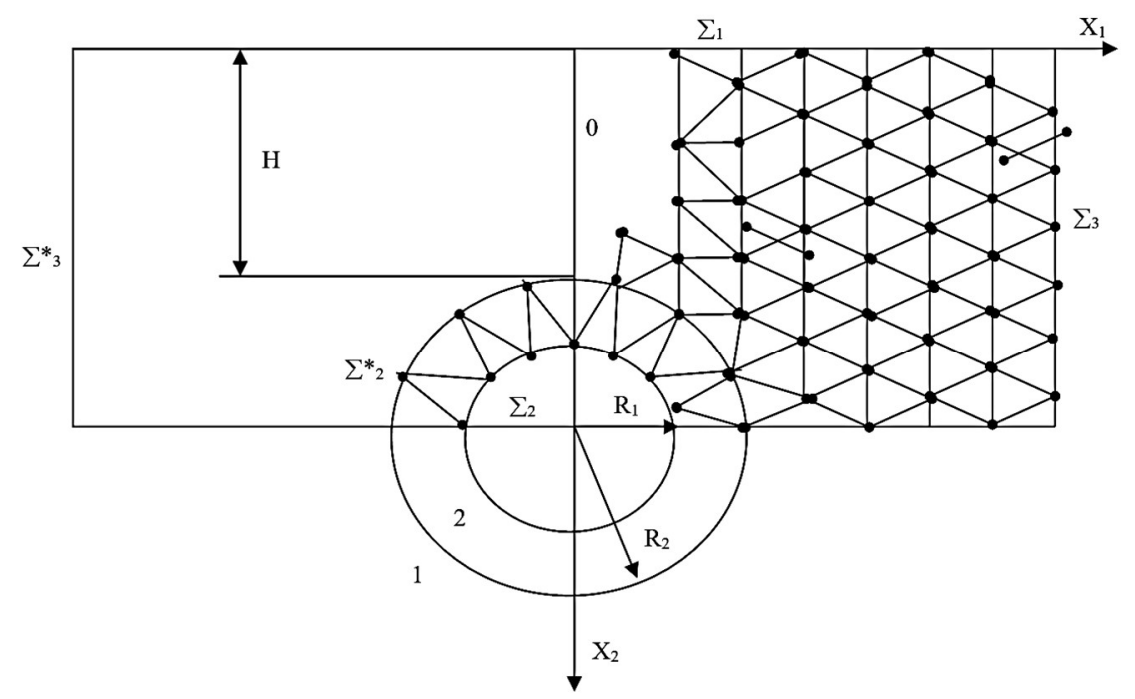

Fig. 1. Calculation scheme.

The equations of motion for the regions $\Omega_{1}$ and $\Omega_{2}$ have the form:

$$
\begin{aligned}
& \frac{\partial^{2} U_{1}}{\partial X_{1}^{2}}+\frac{\partial^{2} U_{1}}{\partial X_{2}^{2}}+\frac{\lambda_{1}+\mu_{1}}{\mu_{1}}+\frac{\partial}{\partial X_{1}}+\left[\frac{\partial U_{1}}{\partial X_{1}}+\frac{\partial V_{1}}{\partial X_{2}}\right]-\frac{\rho_{1}}{\mu_{1}}+\frac{\partial^{2} U_{1}}{\partial t^{2}}=0 \\
& \frac{\partial^{2} V_{1}}{\partial X_{1}^{2}}+\frac{\partial^{2} V_{1}}{\partial X_{2}^{2}}+\frac{\lambda_{1}+\mu_{1}}{\mu_{1}}+\frac{\partial}{\partial X_{1}}+\left[\frac{\partial U_{1}}{\partial X_{1}}+\frac{\partial V_{1}}{\partial X_{2}}\right]-\frac{\rho_{1}}{\mu_{1}}+\frac{\partial^{2} V_{1}}{\partial t^{2}}=0
\end{aligned}
$$

The general area is as follows: $\Omega=\Omega_{1}+\Omega_{2}$;

The following conditions are imposed on the free and contact boundaries:

$$
\begin{gathered}
\sum_{1}\left(X_{2}=R_{2}+H, X=0\right): \sigma_{11}=\sigma_{12}=0, \\
\sum_{2}{ }_{2}\left(X_{1}=R_{1} \cos \theta, X_{2}=R_{2} \sin \theta\right) ; \sigma^{(2)}{ }_{11} \cos \theta=\sigma^{(2)}{ }_{12} \sin \theta=O, \\
\sum_{2}{ }_{2}\left(X_{1}=R_{1} \cos \theta, X_{2}=R_{2} \sin \theta\right) ; \sigma^{(1)}{ }_{11} \cos \theta+\sigma^{(1)}{ }_{12} \sin \theta=\sigma^{(2)}{ }_{12} \sin \theta, \\
\sigma^{(1)}{ }_{11} \cos \theta+\sigma^{(1)}{ }_{22} \sin \theta=\sigma^{(2)}{ }_{12} \cos \theta+\sigma^{(2)}{ }_{22} \sin \theta, \\
U^{(1)}=U^{(2)}, V^{(1)}=V^{(2)} .
\end{gathered}
$$

The movement of $U$ points of the selected area is sought in the form:

$$
\vec{U}(\vec{x}, t)=\vec{U}_{0}(\vec{x}, t) \vec{U} *(\vec{x}, t) .
$$

where

$$
\begin{aligned}
& \left(\begin{array}{l}
U_{0} \\
V_{0}
\end{array}\right)\left(\begin{array}{c}
-A a_{1} \exp \left(-a_{1} X_{2}\right)+b i k \exp \left(-a_{2} X_{2}\right) \\
A a_{1} \exp \left(-a_{1} X_{2}\right)+b i k \exp \left(-a_{2} X_{2}\right)
\end{array}\right) \exp (k x-w t) \\
& a_{1}=w\left(C_{R}^{2}+\rho_{1} \mu_{V}^{-1}\right)^{1 / 2} \\
& a_{2}=w\left(C_{n}^{2} R+\rho_{1}\left(\lambda_{1}+\mu_{V}^{-1}\right)^{1 / 2}\right. \\
& \vec{U}_{0}(x, t)=U_{0} \vec{i}+V_{0} \vec{j}
\end{aligned}
$$


$U_{0}$ and $V_{0}$ - displacements of the half-space surfaces under the influence of the Rayleigh wave, $a$ and $b$ are arbitrary constants that are determined from the boundary conditions (2):

$$
\left.\begin{array}{l}
\frac{\partial u_{1}}{\partial x_{3}}+\frac{\partial u_{3}}{\partial x_{1}}=0, \frac{\partial u_{2}}{\partial x_{3}}+\frac{\partial u_{3}}{\partial x_{2}}=0 \\
\lambda\left[\frac{\partial u_{1}}{\partial x_{1}}+\frac{\partial u_{2}}{\partial x_{2}}+\frac{\partial u_{3}}{\partial x_{3}}\right]+2 \mu \frac{\partial u_{2}}{\partial x_{2}}=0
\end{array}\right\} \text { at } x_{2}=0 .
$$

The functions $\mathrm{U} *(\mathrm{x}, \mathrm{t})$ are determined by the following variational equation:

$$
\begin{aligned}
& -\int_{v} \sigma_{i j}^{*} \delta \varepsilon_{i j} d V+w^{2} \int_{v} \rho \vec{V} \vec{\delta} U^{\varkappa} d V-i w \int_{S_{5}+S_{5}+S_{6}} \sigma_{i j}^{*} V_{j} \delta U_{j} d s+\int_{v_{1}+v_{2}} f \delta U d V+\int_{S_{2}} \rho \delta U d S=0 \\
& \left(\begin{array}{l}
f_{0} \\
f_{0}
\end{array}\right)=\otimes\left(X_{2}\right)\left\{\begin{array}{l}
\sin w\left[t=\frac{X_{1}}{C_{R}}\right] \\
\cos w\left[t=\frac{X_{1}}{C_{R}}\right]
\end{array}\right\}, \quad \quad \mathrm{P}=P\left(X_{2}\right)\left\{\begin{array}{l}
\sin w\left[t=\frac{X_{1}}{C_{R}}\right] \\
\cos w\left[t=\frac{X_{1}}{C_{R}}\right]
\end{array}\right\},
\end{aligned}
$$

and satisfy the radiation condition at $X>S_{5}{ }^{*}$. Here $f$ are external forces.

The discretization of the area under consideration is carried out using triangular elements. The procedure in the first chapter is also repeated here. The problem is reduced to solving a system of inhomogeneous algebraic equations. The system of inhomogeneous complex algebraic equations was solved by the Gauss method, with the following initial data:

$$
\mathrm{v}_{1}=0.20, \mathrm{v}_{2}=0.33, H / R=2.10,15 . E_{1} / E_{2}=0.1 \text {. }
$$

\section{Results and discussions}

\subsection{Analysis}

The calculation results are shown in Fig. 2. The data presented by the solid line were obtained in the study [1], and those marked with asterisks were obtained by our method, with different partitions of the computational domain $(1-N=45,2-N=78,3-N=144)$. Comparison of the results obtained by the analytical method and by the finite element method shows that the maximum difference between them, for hoop stresses, reaches 10-15\%. In Fig. 3, similar results were obtained for $H / \alpha=3$ and 4 , respectively $\left(\theta=90^{\circ} ; \theta=180^{\circ}\right)$. It can be seen that with an increase in the depth of occurrence, the stress decreases noticeably.

In a similar formulation, the problem of vibrations of rectangular bodies in an elastic (viscoelastic) medium is solved.

Now let us investigate the process of vibration propagation in deformable media from a rectangular body (Fig. 4). We apply a harmonic load to a rectangular body, then we get an algebraic system of complex equations:

$$
\left\{[\mathrm{K}]+\mathrm{i} 2 \pi \omega[\mathrm{C}]-4 \pi^{2} \omega^{2}[\mathrm{M}]\right\}\{\mathrm{U}\}=\{\mathrm{P}\} .
$$

Here $\{U\}$ is the vector of complex amplitudes of system oscillations; $\{P\}$ - vector of external load amplitudes, $\omega$ - frequency of external load. A computer calculation was performed for 1020 triangular finite elements. Fig. 4 shows the moduli of curves of the amplitudes of displacements of vertical vibrations of the body under the influence of a load with a unit amplitude. In this case, the following initial data were taken:

$$
\mathrm{v}_{1}=0.36, \mathrm{v}_{2}=0.20, \rho_{1} / \rho_{2}=0.85, E_{1} / E_{2}=0.01, H / \alpha=2.0 \text {. }
$$

It can be seen from Fig. 4 that with an increase in frequency and distance, the amplitudes of displacement decrease. Consequently, during the diffraction of plane harmonic waves in the half-space, surface Rayleigh waves always appear. Their amplitude on the surface $X 2=0$ 
depends on the depth of the tunnel. As $H$ grows, it decays exponentially. The analysis of this decision allows us to draw a practical conclusion. Extensive underground structures in seismically hazardous areas act as generators of surface waves during earthquakes, converting partially incident seismic waves into secondary surface waves. The presence of such inhomogeneities on the path of a seismic wave affects the formation of a Rayleigh surface wave. The cross-sectional shape of cylindrical bodies is not of fundamental importance for this phenomenon.

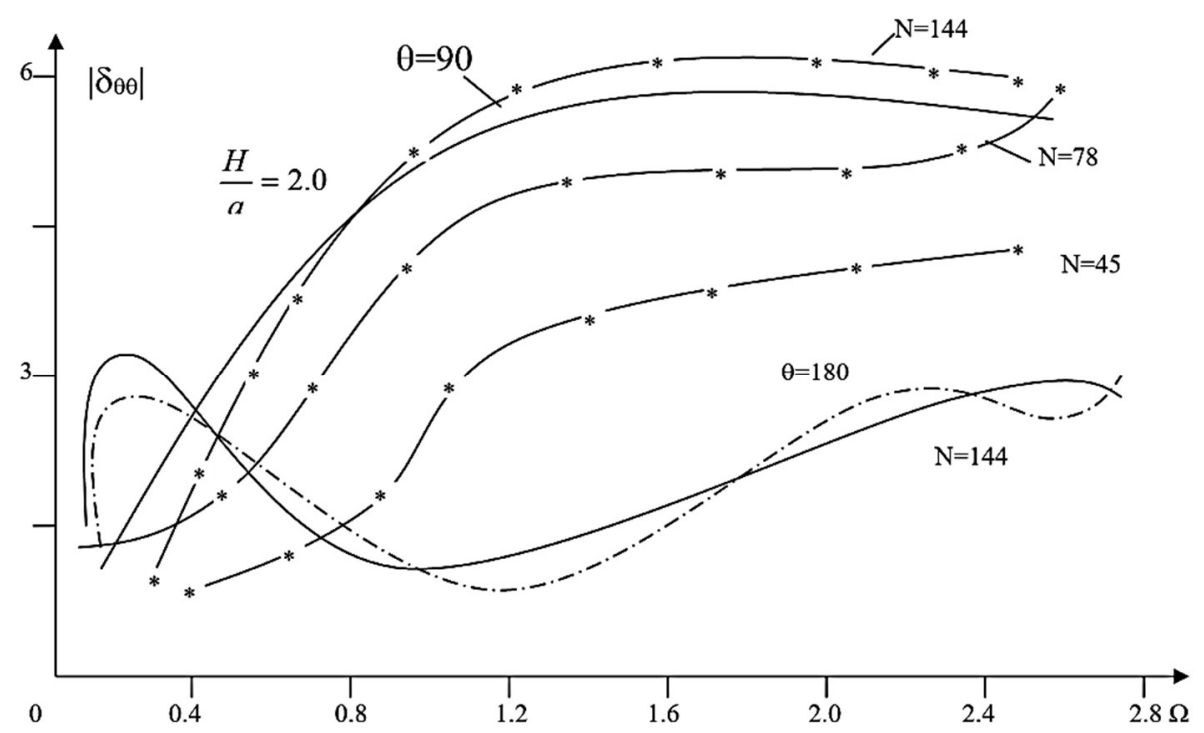

Fig. 2. Hoop voltage variation depending on the frequency of external disturbances.

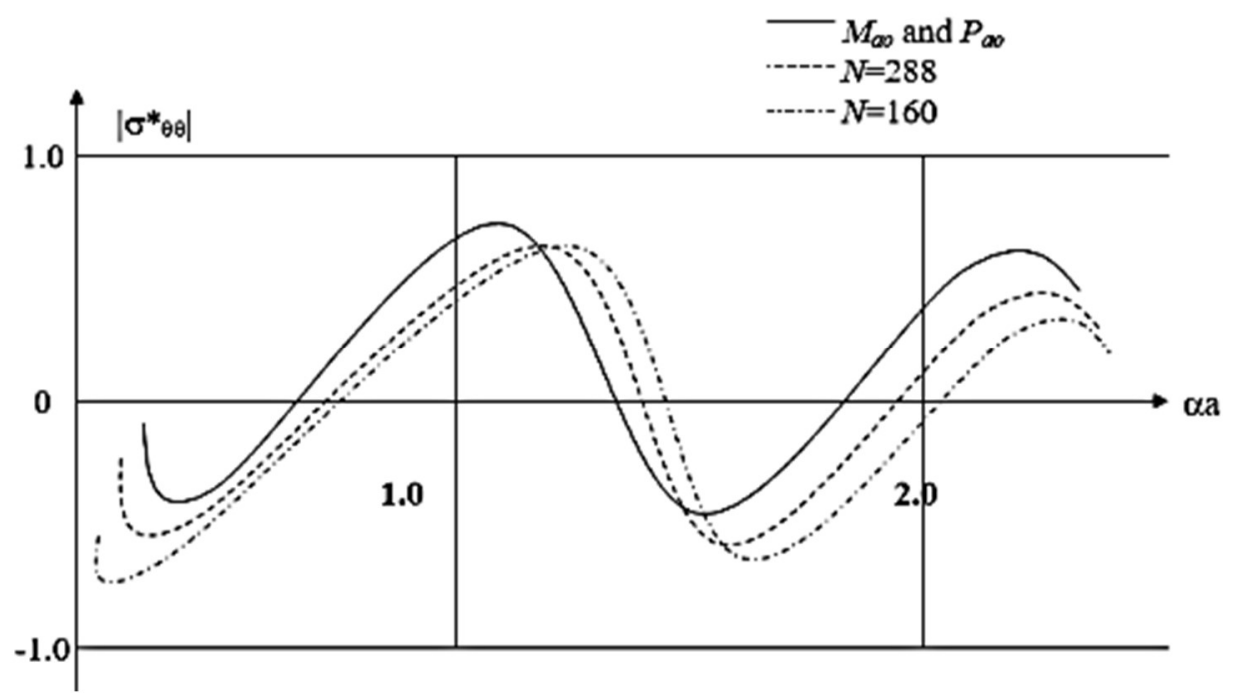

Fig. 3. Change in the hoop voltage depending on the frequency of external disturbances.

The horizontal and vertical components of the pressure force are related by their ratio corresponding to the empirical coefficient $R_{v}$. If the vertical component of the soil pressure force on the horizontal plane of the section at any depth $Z$ from its outer surface is equal to $\sigma_{\mathrm{v}}$, then the normal component of the pressure force on the vertical sliding plane of the elastic 
element is $K_{r}, \sigma_{\mathrm{v}}$ (Fig. 5-8). Solving the equilibrium equations for the distribution of the vertical component of the pressure forces per unit length of the section, we obtain:

$$
\sigma_{v}=\frac{b(y-2 c / b)}{2 k_{r} \operatorname{tg} \theta}\left[1-e^{-2 R_{r}(z / \sigma) \operatorname{tg} \varphi}+q e^{-k_{r}(3 z / b) \operatorname{tg} \theta}\right] .
$$

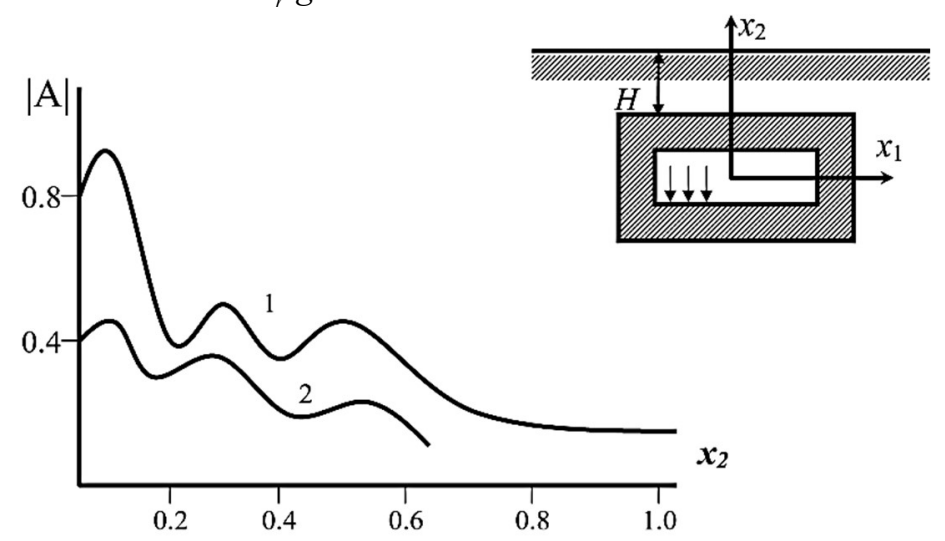

Fig. 4. Change in displacements depending on the distance $\mathrm{x}_{2}$ at: $1 . w=0.2 ; 2 . w=0.8$.

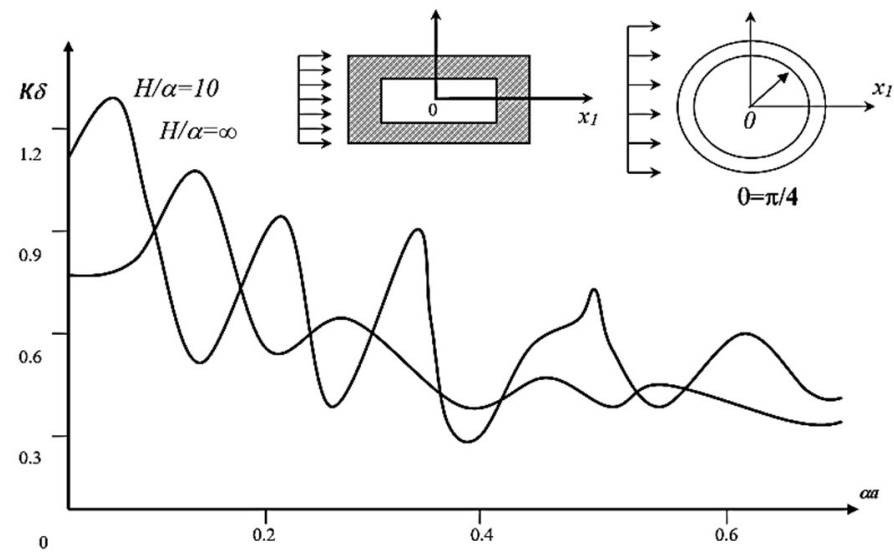

Fig. 5. Dependence of $\sigma_{0}^{*}$ on the free contour of the body on the values of the parameter $\alpha / \lambda$.

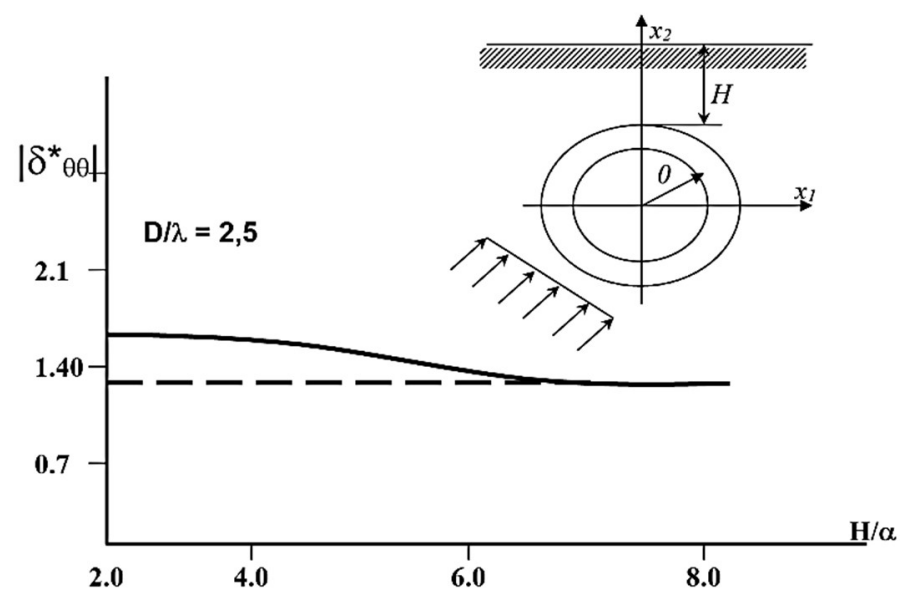

Fig. 6. Dependence of $\delta_{\theta \theta}{ }^{*}$ on the free contour of the body on the values of the parameter $H / \alpha$. 


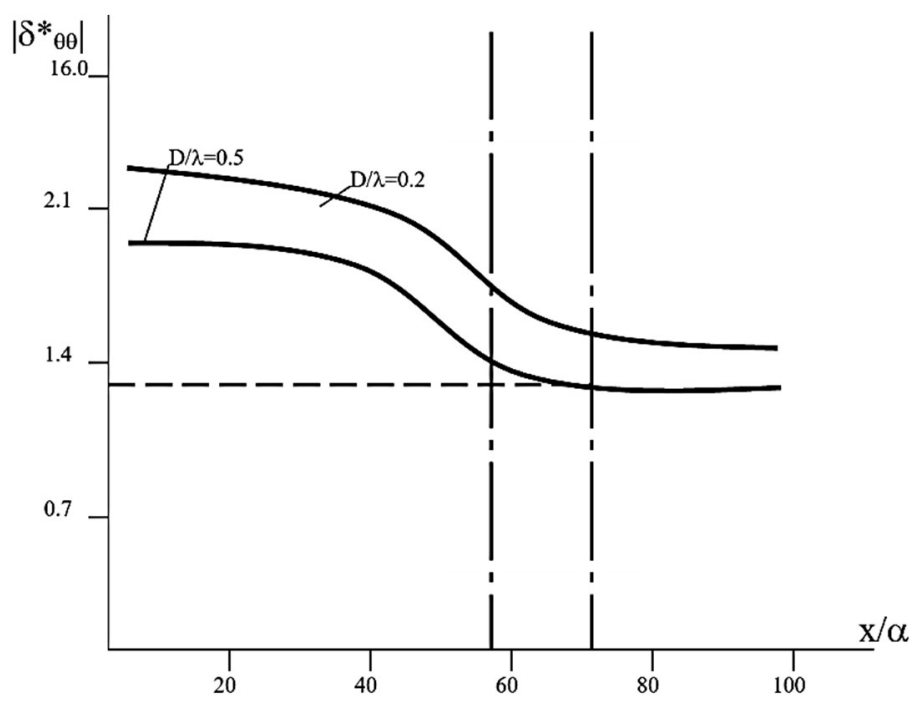

Fig. 7. Dependence of $\delta \theta \theta^{*}$ on the free contour of the body on the values of the parameter $X / \alpha$.

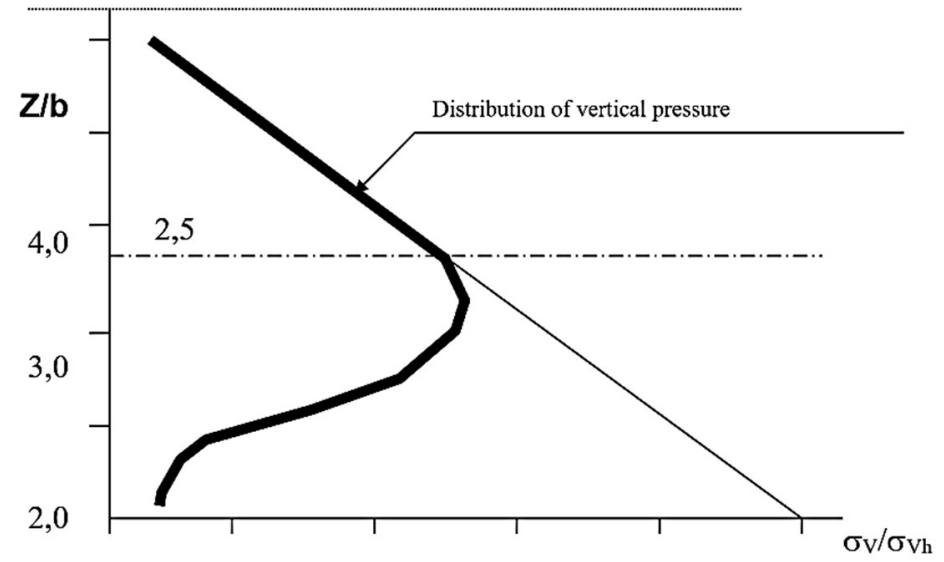

Fig. 8. Results of experiments conducted by Tersagi.

For a soil such as dry sand that does not possess special forces $(c=0)$, the vertical component of the pressure force $\sigma_{v w}$ on an elastic strip for a section at a great depth of the soil approaches its maximum value: with a soil thickness of more than $2.5 \mathrm{~b}$ elastic $\sigma_{v w}=b_{y} /(2 \mathrm{k}$, $\operatorname{tg} \theta$ ) mixing of the rectangular insert does not affect the stress distribution structure for a given soil depth. In [4] it is indicated that the dimensions of the structure, the area of its crosssection in the plane, depending on the depth of its location, affect the arch factor. In addition, the essential importance of the rigidity of the structure is noted and the following expression is proposed as a factor of arch $[5,6]$ (if the pressure of the «free field» of the soil at the depth of the shifting element is equal to $P_{v}$, and the real pressure at the interface «elastic elementsoil» is $P_{t}$, then the proportion of the load transmitted from the structure is $1-P_{t} / P_{v}$. This expression is called the «arch factor»):

where

$$
\left(1-A / A_{0}\right)^{1-A}=e^{-n \Omega}
$$

$$
\Omega=A_{g}\left(M_{3} / P_{v}\right) \delta ; R=A_{g}\left(M_{3} / P_{t}\right) \delta
$$

$A_{g}$ - geometric factor; $M_{3}$ - the secant modulus of elasticity of the soil, determined by compression texturing; $\delta$-displacement of the structure relative to the free-soil field. It has 
been established that the phenomena of active and passive arches can also be observed in the environment of a bound soil, manifesting at a distance of one diameter above the outer surface of the inclusion. Also, the existence of types of reaction of the bound soil to the applied pressure has been established. Until now, the phenomenon of arching, which occurs under loads acting on the upper surface of an elastic structure, has been considered. But, if the underground structure is in the form of a horizontal tunnel, it is necessary to determine the forces acting on its walls, since they can cause deformation of the lower base surface of the tunnel.

In the dynamic calculation of arches, varying degrees of dependence on wavelength and soil properties appear. The presence of the arched phenomenon for bodies in a half-space is numerically established. Analysis of the numerical results makes it possible to evaluate the phenomenon of archedness by the following inequality:

$$
\frac{Z}{b}>y(0,5 e)^{\lambda / b}+Z_{0}
$$

where $\gamma$ is a constant parameter $(0<\gamma<1) ; Z_{0}$ is the value of the arch effect for a static task; $b$ - geometric parameter; $\lambda$ is the wavelength.

\section{Conclusion}

Based on the research carried out, the following conclusions were made:

1. On the basis of the variational method (FEM), the stress-strain state of a cylindrical layer (hole) under the action of harmonic waves is investigated. It was found that the maximum stress concentration is allowed at long waves. Scattering effects become dominant with decreasing wavelength.

2. The oscillation of an elastic half-space containing circular and rectangular obstacles under the influence of harmonic waves has been investigated. The dissipation of the area under consideration is carried out using triangular elements. It has been established that the depth of placement affects the stress-strain state of the body. The concentration of stress with increasing depth and wavelength approaches the static value of stress.

3. A calculation method has been developed for studying the natural vibrations of piecewise-homogeneous deformable systems in an elastic medium, taking into account the internal and wave energy dissipation.

4. The effect of arching was established under the dynamic impact of underground structures.

\section{References}

1. Y.H. Pao, C.C. Mow. The diffraction of elastic waves and dynamic stress concentrations. N.Y.: Crane Russak and Co 694 (1973).

2. H. Huang, Y.P. Lu, Y.F. Wang. Transient interaction of spherical acoustic waves a cylindrical elastic shell and its internal multi-degree-of-freedom mechanical systems, J. Acoust. Soc. America 56 1, 4-10 (1974).

3. S.P. Howe, W. Goldsmiths, J.L. Sackman. Macroscopic static and dynamical mechanical properties of Yule marble, Experimental Mechanics 14 9, 337-346 (1974).

4. D.A. Howells. Tunnels in earthquake areas, Tunnels and tunneling, 437 (1972).

5. Y. Kimura. Function of Tokio port tunnel is for developing Tokio by area, Civil Engineering in Japan 15, 58-66 (1976).

6. T.R. Kuesel. Earthquake design criteria for subways, J. Str. Div. 95 6, 1213-1231 (1966). 